Topol ogi cal for mat i on of a mul t i pl y charged vortex i $n$ the $\mathrm{Rb}$ Bose- Ei nst ei $\mathrm{n}$ condensate: Effect i veness of the gravi ty compensati on

\begin{tabular}{|l|l|}
\hline 著者 & $\begin{array}{l}\text { KUNAKURA M, H ROTAN T, OKANO M TAKAHASH Y, } \\
\text { YABUZAK T }\end{array}$ \\
\hline $\begin{array}{l}\text { j our nal or } \\
\text { publ i cat i on ti t l e }\end{array}$ & Physi cal Revi ew A \\
\hline vol une & 73 \\
\hline number & 6 \\
\hline page range & 63605 \\
\hline year & $2006-06$ \\
\hline URL & ht t p: //hdl . handl e. net /10098/1783 \\
\hline
\end{tabular}

doi: 10.1103/PhysRevA.73.063605 


\title{
Topological formation of a multiply charged vortex in the Rb Bose-Einstein condensate: Effectiveness of the gravity compensation
}

\author{
M. Kumakura, ${ }^{1,2,3, *}$ T. Hirotani, ${ }^{1}$ M. Okano, ${ }^{1}$ Y. Takahashi, ${ }^{1,3}$ and T. Yabuzaki ${ }^{1, \dagger}$ \\ ${ }^{1}$ Department of Physics, Graduate School of Science, Kyoto University, Kyoto 606-8502, Japan \\ ${ }^{2}$ PRESTO, JST, 4-1-8 Honcho Kawaguchi, Saitama 332-0012, Japan \\ ${ }^{3}$ CREST, JST, 4-1-8 Honcho Kawaguchi, Saitama 332-0012, Japan
}

(Received 14 September 2005; revised manuscript received 6 May 2006; published 6 June 2006)

\begin{abstract}
In a Bose-Einstein condensate of ${ }^{87} \mathrm{Rb}\left(F=2, m_{F}=2\right)$ atoms we have topologically created a quantized vortex with a charge of 4 by reversing the magnetic field of the trap. Experimental conditions of reversal time and initial magnetic field strength for the successful vortex creation were restricted within narrower ranges, compared to those in the case of the ${ }^{23} \mathrm{Na}$ condensate. The experimental difficulty was explained in terms of a non-negligible gravitational sag arising from its large atomic mass. We have successfully stabilized the vortex formation by compensating gravity with a blue-detuned laser beam.
\end{abstract}

DOI: 10.1103/PhysRevA.73.063605

PACS number(s): 03.75.Kk, 03.75.Lm, 67.90.+z

\section{INTRODUCTION}

The motion of a quantum fluid, like superfluid liquid $\mathrm{He}$, electron pairs in a superconductor, or Bose-Einstein condensates (BECs) in atomic dilute gases is described by a macroscopic wave function. The velocity field $v(r)$ of the fluid is determined by the gradient of the phase $\theta(r)$ of the wave function, so that the velocity field $v(r)$ is irrotational $(\nabla \times v(r)=\nabla \times \nabla \theta(r)=0)$ and the rotational motion can be obtained only in the form of a quantized vortex [1]. So far the quantized vortices have been observed, for example, as vortex lines in superfluid $\mathrm{He}$ [2] and flux lines in superconductors [3]. It is well known that the quantized vortex plays an important role in the dynamics of the quantum fluid.

BECs in dilute atomic gases, which were realized in this decade, are attractive systems for studying the quantum fluid due to their high controllability and good optical accessibility $[1,4]$. The quantized vortex has been created also in such atomic systems [5-12], and novel phenomena such as a vortex array [6-10] or a giant vortex [11] have been observed. Vortices in the gaseous BECs have been created by the following four methods: dynamical phase imprinting [5], optical spoon stirring [6,7], a rotating asymmetric trap [8-11], and topological phase imprinting [13]. Among these methods, only the last one can produce a multiply charged vortex, which has never, to our knowledge, been observed in traditional systems like superfluid ${ }^{4} \mathrm{He}$. The winding number of the vortex obtained by this method is two times the quantum number of the atomic angular momentum. The vortices with the winding number 2 or 4 were recently achieved in BECs of $\mathrm{Na}$ in the ground $F=1$ or 2 states, respectively $[14,15]$.

The multiply charged vortex is energetically unstable towards splitting to plural vortices with smaller winding numbers [16]. This decay process of the multiply charged vortex

\footnotetext{
*Present address: Graduate School of Engineering, University of Fukui, Bunkyo, Fukui 910-8507, Japan, Electronic address: kumakura@apphy.fukui-u.ac.jp

†Present address: Osaka Electro-Communication University, Neyagawa, Osaka 572-8530, Japan.
}

is driven coherently by atomic interactions, which is called dynamical instability [17]. The splitting of a doubly charged vortex to two singly charged vortices was observed in the $\mathrm{Na}$ BEC [18], but its lifetime observed in this experiment showed a monotonous increase with the increase of the interatomic interaction strength, contrary to the theoretical result which predicted the periodic behavior [17]. To understand this discrepancy, detailed experimental study on a multiply charged vortex in other atomic species or with a higher winding number is important. One good candidate for such experiments is a rubidium atom, which is widely used in the gaseous BEC experiment and whose characteristics are well understood. In this alkali atomic species we can form a vortex with a winding number of 2 or 4 in the same way as in $\mathrm{Na}$, so that we can compare the dynamical instability between these superfluids. In addition, due to the small spin exchange collision rate in $\mathrm{Rb}$ [19], differing from $\mathrm{Na}$, it is possible to make a binary mixture of BECs with different winding numbers by using atoms in the $F=1$ and 2 ground hyperfine states, which probably enable us to explore novel phenomena on superfluidity.

In this paper, we report on our generation of a quadruply charged vortex in the ${ }^{87} \mathrm{Rb} \mathrm{BEC}$, which was confined in an Ioffe-Pritchard magnetic trap. Especially in the case of the quadruply charged vortex, it is predicted that the splitting pattern of the vortex can be changed by the symmetry of the trap potential distortion [17], which has not yet been observed. For experimentally studying such coherent dynamics of a superfluid, it is important to realize the stable formation of the vortex. The ${ }^{87} \mathrm{Rb}$ atom constituting the BEC was in the $F=2, m_{F}=2$ state, and we have topologically created a quadruply charged quantized vortex by reversing the axial magnetic field. We found, however, that compared with the case of the Na BEC [14], experimental conditions, such as the field reversal time and initial magnetic field strength, for the successful creation were restricted within narrower ranges for $\mathrm{Rb}$, and the observed holding time of about $1 \mathrm{~ms}$ was also much shorter. To understand what causes such big difference between $\mathrm{Rb}$ and $\mathrm{Na}$, we have carried out elementary numerical calculation for the center-of-mass motion of the $\mathrm{BEC}$, and found that the gravitational sag of the BEC from 


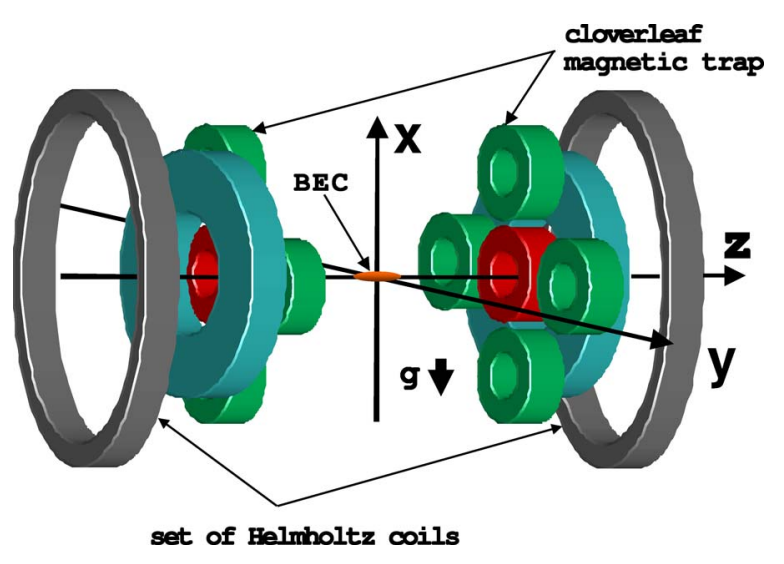

FIG. 1. (Color online) Experimental setup. The magnetic trap consists of 12 coils placed in the cloverleaf configuration. The magnetic field at the trap center is directed along the $z$ direction, and its strength is about $0.4 \mathrm{G}$, which is reversed by applying an additional axial magnetic field with an external set of Helmholtz coils for the topological vortex formation. The gravity is in the $-x$ direction.

the symmetry axis of the magnetic field plays an important role in the case of $\mathrm{Rb}$. Based on this calculation, we have removed the experimental instability in the vortex creation by compensating the gravitational field with a blue-detuned laser beam, which is weakly focused below the trap center.

\section{EXPERIMENT: TOPOLOGICAL VORTEX CREATION}

Our experimental apparatus for making a BEC in $\mathrm{Rb}$ was described in detail previously [20]. ${ }^{87} \mathrm{Rb}$ atoms in the $F=2$ state were optically collected and cooled by a doublemagneto-optical trap system [21], and were transferred into a magnetic trap (Fig. 1) after the polarization gradient cooling and optical pumping. The magnetic trap was constructed in the cloverleaf configuration [22]. The $\mathrm{Rb}$ atoms in the $F=2, m_{F}=2$ state were confined in a magnetic trap and BEC was achieved by rf evaporative cooling.

The magnetic field strength at the trap center $B_{0}$ was $0.4 \mathrm{G}$, which was the minimum value in the trap, and its direction was along the $z$ direction. The magnitude of the radial magnetic field was approximately linear, and that of the axial field was nearly harmonic. The axial and radial magnetic field gradients $B_{z}^{\prime \prime}$ and $B_{r}^{\prime}$ were about $140 \mathrm{G} / \mathrm{cm}^{2}$ and $180 \mathrm{G} / \mathrm{cm}$, respectively. The axial and radial potential energy curves were approximately harmonic in the vicinity of the BEC, and the axial and radial trap frequencies $\omega_{z}$ and $\omega_{r}$ were $2 \pi \times 15 \mathrm{~Hz}$ and $2 \pi \times 360 \mathrm{~Hz}$, respectively. The number of atoms in the BEC was about $3 \times 10^{5}$, and the chemical potential was about $140 \mathrm{nK}$. The axial and radial diameters of the BEC were approximately 120 and $6 \mu \mathrm{m}$, respectively.

The magnetic field direction around the symmetric axis (that is, the $z$ axis in Fig. 1) is schematically shown in Fig. 2. The direction of the radial magnetic field $B_{r}$ at the position $(x, y)$ was opposite to that at $(-x,-y)$. In the vicinity of the $\mathrm{BEC}$, the magnitude of the radial magnetic field $\left|B_{r}\right|$ was estimated to be less than $90 \mathrm{mG}$ from the diameter of the (a)

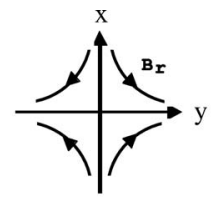

(b)

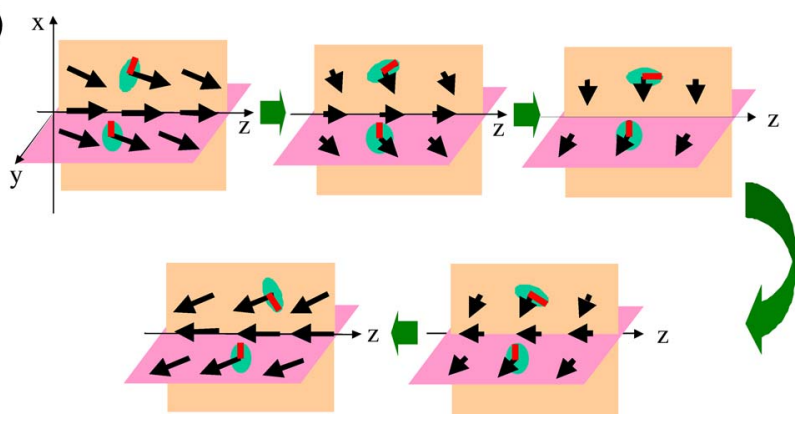

FIG. 2. (Color online) Schematic diagram of the topological vortex formation. (a) The magnetic field direction in the $x-y$ plane, which remains constant. (b) The axial magnetic field is gradually reversed by applying an additional axial magnetic field. Each arrow represents the magnetic field direction, and the direction of each atomic spin follows the change of the local field direction adiabatically. The needles on the small disks indicate the atomic phases. Initially the atomic phase is identical all over the BEC. During the axial field reversal, because the direction of the rotation of the atomic spin is different depending on the spatial position of the atom, the different Berry phase is imprinted to the atom depending on its position, which results in the quantized vortex.

BEC. Therefore, initially the axial magnetic field along the $z$ axis $(\sim 0.4 \mathrm{G})$ dominantly determined the magnetic field direction in the vicinity of the BEC. By applying an additional homogeneous magnetic field along the $-z$ direction, the axial magnetic field was linearly decreased and the field direction was approximately reversed in about $2.2 \mathrm{~ms}$ (Fig. 3). During the field reversal the Larmor precession frequency of the

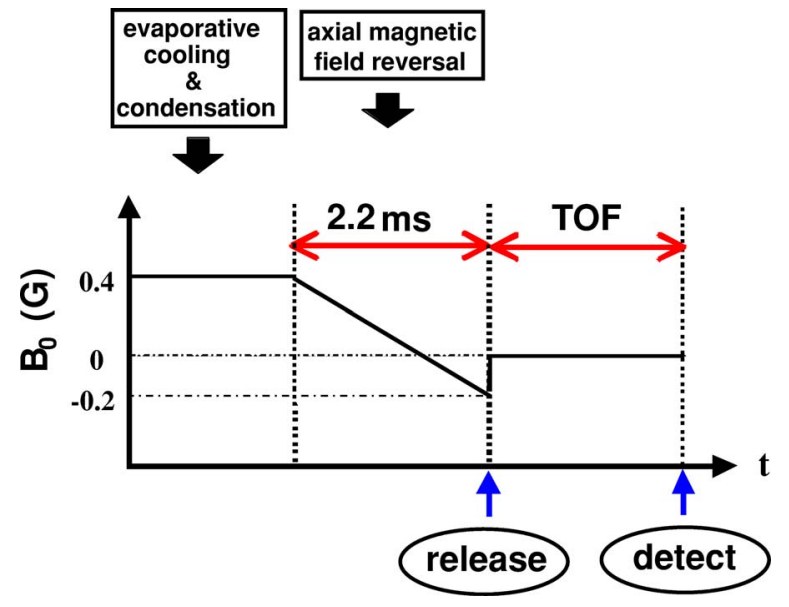

FIG. 3. (Color online) Typical time sequence of the axial magnetic field reversal. The axial magnetic field at the trap center $B_{0}$ was reversed typically from 0.4 to $-0.2 \mathrm{G}$ in a time of $2.2 \mathrm{~ms}$ by applying an additional axial magnetic field with an external set of Helmholtz coils. A created vortex was observed by the absorption imaging method after releasing the BEC from the trap. The timeof-flight was typically $13 \mathrm{~ms}$. 


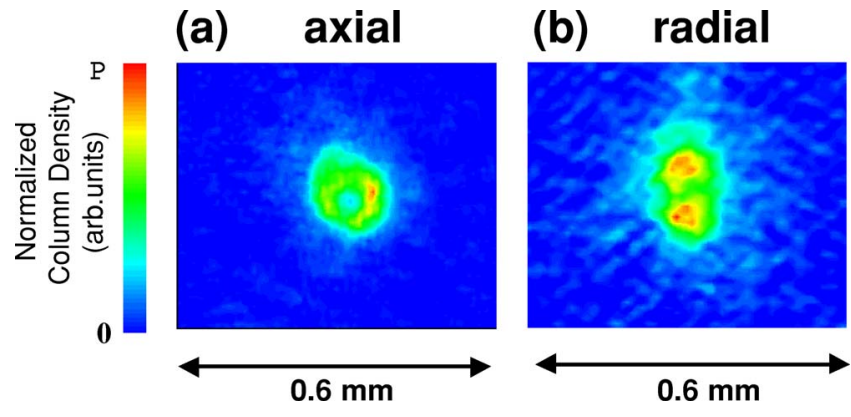

FIG. 4. (Color online) Images of the BEC with a quantized vortex observed from (a) the axial direction (the $z$ direction) and (b) the radial direction (the $y$ direction). In (a) a created vortex is seen as a hole in the atom density, while in (b) a vortex line is seen as a linear depletion along the horizontal axis (the $z$ axis). The resolution of the image is about $16 \mu \mathrm{m}$. These images were observed after a time-of-flight of $13 \mathrm{~ms}$ by the absorption imaging.

atomic spin was estimated to be higher than $1.3 \mathrm{kHz}$ for the atoms at a distance longer than $0.05 \mu \mathrm{m}$ (about onehundredth of the BEC radius) from the $z$ axis. Thus, for almost all atoms in the BEC, the Larmor precession frequency was so large that the atomic spin followed adiabatically the slow change of the magnetic field direction and was oriented as shown in Fig. 2 depending on the spatial position. Finally, the spin direction was approximately reversed following the magnetic field reversal. Through the field reversal we imprint the phase difference in the BEC, which arises from the spatial difference of the Berry phase carried by the atomic spin.

With the above-mentioned experimental setup and procedure, the vortex was observed as depletion in the atomic density distribution of the BEC. In Fig. 4, we show the atomic density profile obtained after a time of flight (TOF) of $13 \mathrm{~ms}$, observed as the absorption image along the radial and axial directions. The BEC was released from the potential by turning off the magnetic field. To retain the atomic spin orientation, the quadrupole radial field was turned off $100 \mu$ s before turning off the axial field. The frequency of the probe laser beam was resonant to the $5{ }^{2} S_{1 / 2}(F=2) \rightarrow 5{ }^{2} P_{3 / 2}(F=3) \quad(\lambda=780 \mathrm{~nm})$ transition, and the resolution of the imaging was about $16 \mu \mathrm{m}$. The atom number contained in the BEC was typically $2 \times 10^{5}$, and the width (FWHM) was about $150 \mu \mathrm{m}$. As seen in the image along the axial direction [Fig. 4(a)], the vortex was observed as the apparent circular depletion in the atomic density distribution. On the other hand, in the image along the radial direction [Fig. 4(b)], the vortex line was seen as linear depletion along the $z$ direction. The depletion could be observed even at a shorter flight time, although the size was smaller and the depth was shallower because of the image resolution.

The width (FWHM) of the observed vortex was about $74 \pm 20 \mu \mathrm{m}$, which was in good agreement with a theoretical value estimated as follows. The uncertainty was estimated from the pixel size of the CCD camera and the uncertainty in the amplification factor of the imaging system. It also contains the uncertainty of about $5 \mu \mathrm{m}$ caused by the misalignment of the probe beam from the vortex axis. In our case the BEC was confined in an anisotropic trap with a cigar shape.
In such a case the size of the vortex was determined from the atomic density $n$ even after the free expansion following the release [23]. In terms of the healing length $\xi=(8 \pi a n)^{-1 / 2}$, where $a$ is the $s$-wave scattering length, the width (FWHM) of a vortex is calculated to be $1.7 \xi, 3.2 \xi, 4.7 \xi$, and $6.1 \xi$ for the winding number of $1,2,3$, and 4 , respectively, under the assumption that the atomic density $n$ is constant in the region far from the vortex center [1]. Therefore, if we assume that the atomic density $n$ can well be approximated to be the measured mean density of the BEC, the width (FWHM) of a quadruply charged vortex is estimated to be $92 \mu \mathrm{m}$ for our experimental condition. This value agrees well with the observed one. From this result and the topological feature of this vortex formation method, we concluded that the winding number of the created vortex was 4 .

This conclusion was further confirmed by observing the atomic population distribution in the magnetic sublevels of the ground hyperfine $F=2$ state. If the atomic spin was not reversed completely, the magnetic sublevels with $m \neq 2$ were populated substantially and the vortex with a winding number of $m+2$, which should be less than 4 , could be created [15]. To check this possibility we made the Stern-Gerlach measurement of the BEC with the vortex, and confirmed that almost all the population was transferred to the magnetic sublevel $m=2$.

The observed vortex gradually diminished in a holding time of about $1 \mathrm{~ms}$, which was much shorter than the value ( $\sim 7 \mathrm{~ms})$ observed in the Na BEC experiment [14]. Definite evidence of splitting of the vortex could not be observed in the present experiment.

For the field reversal from 0.4 to $-0.2 \mathrm{G}$, the vortex was generated only in ranges of the field reverse time $T_{\text {rev }}$; $2.0 \mathrm{~ms} \leq T_{\text {rev }} \leq 2.4 \mathrm{~ms}$ and $4.0 \mathrm{~ms} \leq T_{\text {rev }} \leq 4.3 \mathrm{~ms}$, which were much narrower than those in the $\mathrm{Na}$ BEC experiment. As described in Sec. III, the existence of such narrow ranges for the vortex formation can be explained by the oscillation of the center of mass (hereafter denoted by CM) of the BEC caused by the field reversal under the gravity. To form the vortex in the BEC, it is necessary for the $z$ axis to be in the $\mathrm{BEC}$, when $B_{0}$ is close to the value of $0 \mathrm{G}$. The CM, however, vertically deviates from the $z$ axis due to the gravitational sag. The deviation oscillates during the field reversal, so that this condition is satisfied both when the BEC is moving up and when it is moving down, which explains the existence of these two ranges of $T_{r e v}$.

This range of $T_{\text {rev }}$ appropriate for the stable vortex formation changed as the initial and final values of the minimum magnetic field $B_{0}$ were altered: (i) in the case where $B_{0}$ was reversed from 0.8 to $-0.8 \mathrm{G}$, the appropriate range was that $2.8 \mathrm{~ms} \leq T_{\text {rev }} \leq 3.4 \mathrm{~ms}$; (ii) in the case where $B_{0}$ was reversed from 0.2 to $-0.2 \mathrm{G}$, it was that $2.0 \mathrm{~ms} \leq T_{\text {rev }} \leq 4.0 \mathrm{~ms}$. Even in these ranges the position of the vortex was not always at the center of the BEC and it fluctuated in the BEC each time. In the case where $B_{0}$ was reversed from the value more than $1.0 \mathrm{G}$, we could not create a vortex for any value of $T_{r e v}$.

The vertical oscillation of the BEC might excite the radial breathing mode of the collective oscillation [1]. In our vortex formation, however, we did not observe the collective oscillation. 


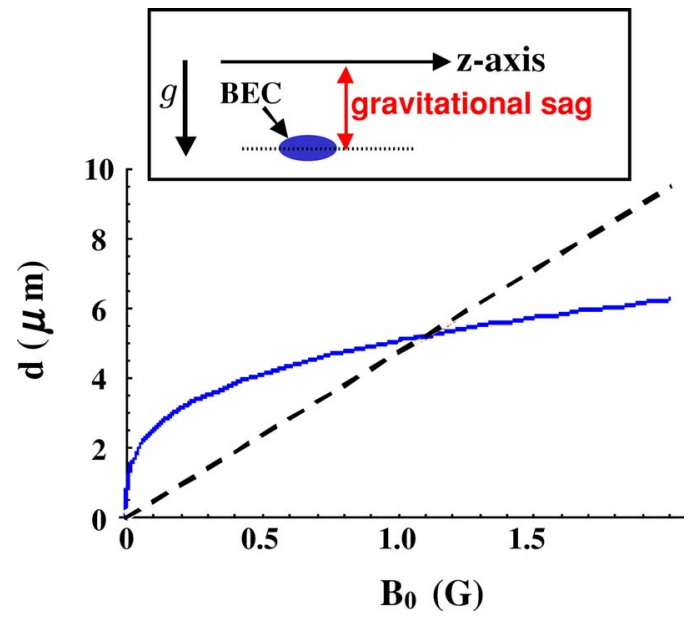

FIG. 5. (Color online) Gravitational sag $d$ (broken line) and $\mathrm{BEC}$ radius (solid line) as a function of the magnetic field strength at the trap center $B_{0}$. The calculation was made for $\mathrm{Rb}$ atoms (atom number of $3 \times 10^{5}$ ) by using the radial magnetic field gradient of $180 \mathrm{G} / \mathrm{cm}$. The BEC radius was calculated under the ThomasFermi approximation.

\section{THEORETICAL DISCUSSION}

The narrow window in $T_{r e v}$ and $B_{0}$ appropriate for the vortex formation can be explained by the gravitational sag of the BEC: because of gravity the vertical position of the BEC confined in the magnetic trap is shifted below the symmetric axis of the magnetic field.

The gravitational sag $d$ is expressed as $g / \omega_{r}^{2}$, where $g$ and $\omega_{r}$ are, respectively, the acceleration of gravity and the radial trap frequency $\left[\omega_{r}=\left\{\gamma /\left(2 m\left|B_{0}(t)\right|\right)\right\}^{1 / 2} B^{\prime}\right.$, where $\gamma$ is the atomic magnetic dipole moment], while the radius of the BEC is expressed as $\left\{2 \mu /\left(m \omega_{r}^{2}\right)\right\}^{1 / 2}$, where $\mu$ and $m$ are the chemical potential of the $\mathrm{BEC}$ and the atomic mass, respectively, under the Thomas-Fermi approximation [1]. For BECs confined with the same $\omega_{r}$, the gravitational sag is the same regardless of their atomic mass. However, the BEC radius depends on the atomic mass. The $\mathrm{BEC}$ radius is smaller for the atoms with larger atomic mass, so that the gravitational sag can exceed the BEC radius for such atoms. If the gravitational sag was negligibly small compared with the BEC radius, then gravity could not affect the vortex formation. On the other hand, if the sag exceeds the radius, the magnetic symmetric axis, on which the vortex should be placed, is displaced from the BEC. In this way we see that gravity strongly affects the vortex formation.

Both the gravitational sag and BEC radius also depend on $B_{0}$ through $\omega_{r}$. In Fig. 5, we show a typical relation between the gravitational sag and $\mathrm{BEC}$ radius, calculated for $\mathrm{Rb}$ atoms under the condition that the total atom number in the $\mathrm{BEC}$ was $3 \times 10^{5}$. We see from Fig. 5 that the gravitational sag exceeds the BEC radius when $B_{0}>1 \mathrm{G}$. Moreover, even when $0.2 \mathrm{G}<B_{0}<1 \mathrm{G}$, it is not negligible compared to the $\mathrm{BEC}$ radius.

The gravitational sag causes vibrations in the CM motion and in the shape of the BEC during the magnetic field reversal. The value of $\omega_{r}$ depends on $\left|B_{0}(t)\right|$, which is altered

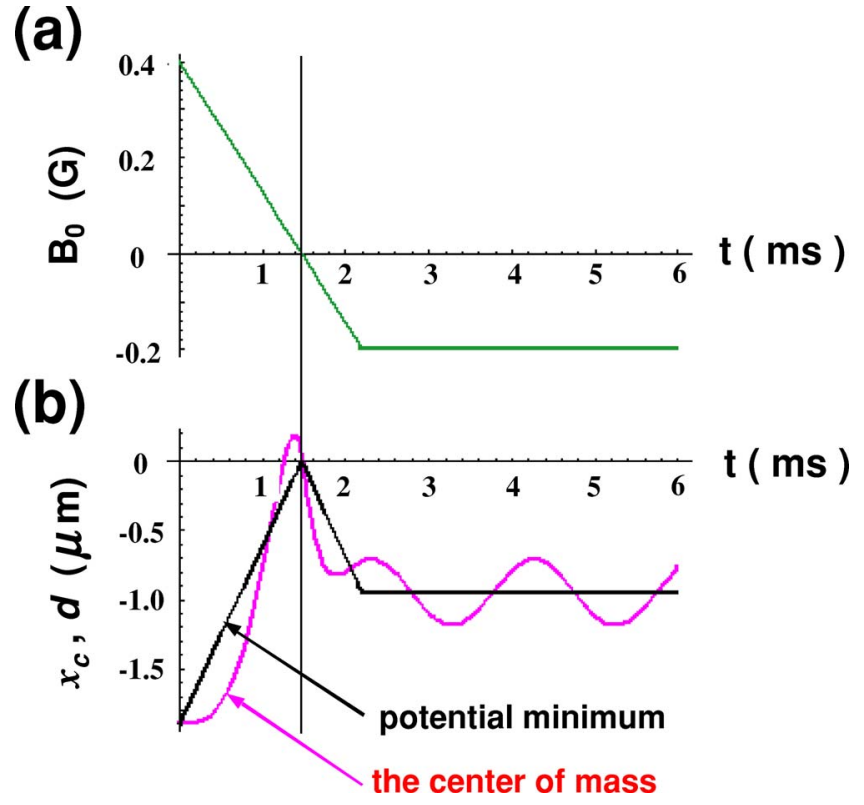

FIG. 6. (Color online) Typical center-of-mass motion during the field reversal. (a) The axial magnetic field is changed from $B_{0}=0.4$ to $-0.2 \mathrm{G}$ in a time of $2.2 \mathrm{~ms}$. (b) The center-of-mass motion and the potential minimum are plotted as a function of time. The center of mass moves up and down around the potential minimum during the field reversal.

during the field reversal, so that the value of the sag, i.e., the position of the trap potential minimum changes also. The $\mathrm{CM}$ of the BEC moves, seeking the new potential minimum and oscillates around it. Through this oscillation the BEC itself was perturbed asymmetrically in the radial direction, which results in the excitation of shape vibration.

To make clear the influence of the gravity on the vortex formation, we have calculated the theoretical path of the $\mathrm{CM}$ motion $x_{c}(t)$ of the ${ }^{87} \mathrm{Rb} \quad \mathrm{BEC}$ by numerically solving the classical equation of motion $M d^{2} x_{c}(t) / d t^{2}=-M g-\partial V\left(x_{c}, t\right) / \partial x_{c}$, where $M$ is the total mass of the BEC, and $V\left(x_{c}, t\right)=M \omega_{r}(t)^{2} x_{c}^{2} / 2$ is a time-dependent potential energy. In Fig. 6(b), we show a typical example of the calculated path and position of the potential minimum where $B_{0}$ is linearly reversed from 0.4 to $-0.2 \mathrm{G}$ in a time of $2.2 \mathrm{~ms}$ as shown in Fig. 6(a).

As seen in Fig. 6(b), before the magnetic field reversal, the gravitational sag is comparable to the $\mathrm{BEC}$ radius $(\sim 3 \mu \mathrm{m})$. After starting the field reversal the BEC begins to move, seeking a new potential minimum. The CM is strongly accelerated toward the potential minimum by the large gradient of the trap potential far from the minimum, which results in its oscillation around the minimum. In this way, the CM motion cannot adiabatically follow the potential minimum. On the other hand, most atomic spins can adiabatically follow the change of the magnetic field. So, even if the CM motion changes the rate of the magnetic field reversal for each atom, the total atomic phase imprinted on each atom during the field reversal is nearly determined by the local magnetic field direction. Therefore, the vortex might be formed along the magnetic symmetry axis at the moment when $B_{0}=0$. To create the vortex in the middle of the BEC, it 


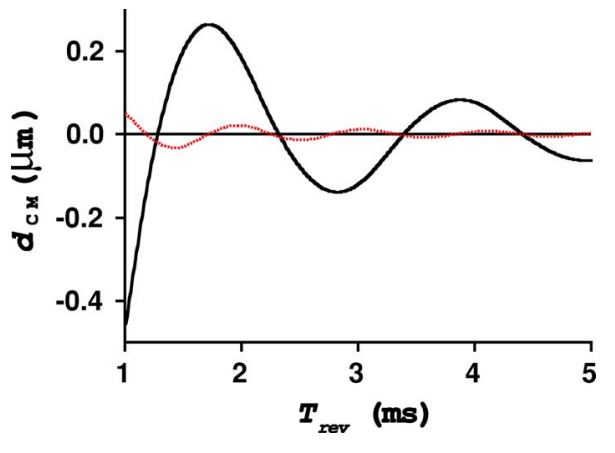

FIG. 7. (Color online) The distance $d_{C M}$ between the CM and the symmetric axis at the moment $B_{0}=0$ as a function of the field reversal time $T_{\text {rev }}$. The distance $d_{C M}$ was calculated in the case where the axial field was changed from $B_{0}=0.4$ to $-0.2 \mathrm{G}$. The solid and dotted lines are for the $\mathrm{Rb}$ and $\mathrm{Na}$ BECs, respectively.

is necessary to place the CM on the symmetry axis at the moment. In the field reversal shown in Fig. 6, this condition is approximately fulfilled in spite of the large CM oscillation during the field reversal. This fact explains the successful vortex formation in our experiment with the similar field reversal.

The distance between the CM and the symmetric axis at the moment $B_{0}=0$, which is denoted by $d_{C M}$ hereafter, is not always negligible. Figure 7 shows the calculated dependence of the distance $d_{C M}$ on the field reversal time $T_{r e v}$. As seen in Fig. 7, the distance $d_{C M}$ oscillates as a function of $T_{r e v}$, and there exist windows of $T_{\text {rev }}$ appropriate for the vortex formation. In the case of the field reversal from 0.4 to $-0.2 \mathrm{G}$, the windows are around the values of $T_{\text {rev }}$ of 2.3 and $3.4 \mathrm{~ms}$, which explains well our experimentally observed windows for the vortex formation. Although our calculation was elementary, the result was confirmed by comparing with more precise quantum theoretical calculation reported recently by Kawaguchi et al. [24].

From our calculation, it was confirmed also that, in the case of $T_{\text {rev }}$ more than $12 \mathrm{~ms}$, the maximum deviation of the $\mathrm{CM}$ from position of the potential minimum was less than $0.1 \mu \mathrm{m}$ during the field reversal, which corresponded to $5 \%$ of the initial gravitational sag and about $3 \%$ of the BEC radius. Therefore, in such slow field reversal, the $\mathrm{CM}$ motion can be considered to be adiabatic. It should be noted, however, that we could not experimentally observe the formation of the vortex even if $T_{\text {rev }}$ was longer than $4 \mathrm{~ms}$. This can be explained in terms of Majorana spin flips occurring during the field reversal. Indeed, in such a condition, we observed a considerable decrease in the atom number of the BEC during the field reversal, as is predicted by the precise theoretical calculation [24]. They predicted also the splitting of the generated vortex during the field reversal, but we could not observe it in the present experiment.

The calculation predicts the vortex formation in another window around $T_{\text {rev }}=1.3 \mathrm{~ms}$, but in our experiment we could not confirm the stable vortex formation around this value. This discrepancy may be due to our experimental condition, in which we could not change the magnetic field linearly in such a short $T_{\text {rev }}$ as assumed in the theoretical calculation.

Figure 6 shows also that the oscillation of the CM continues even after the end of the field reversal. The amplitude of

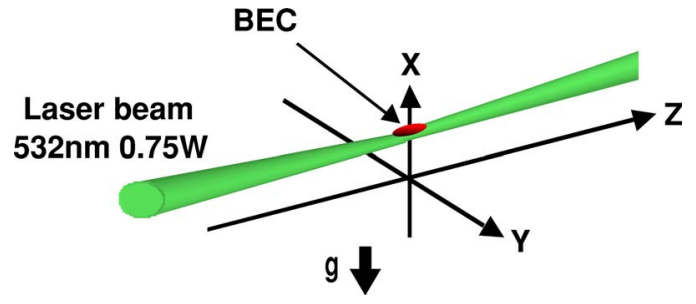

FIG. 8. (Color online) Compensation of the gravitational field by using a blue-detuned laser beam $(532 \mathrm{~nm}, 0.75 \mathrm{~W})$. The beam is softly focused $54 \mu \mathrm{m}$ below the BEC, whose waist size is about $53 \mu \mathrm{m}$. The vertical potential gradient generated by the laser beam is set nearly $-m g$ at the position of the BEC, so as to compensate the gravitational field.

oscillation strongly depends on $T_{\text {rev }}$, and the oscillation after the field reversal cannot be removed under the condition of the successful vortex formation. This oscillation can excite internal vibrational modes and/or vibration of a vortex line, which may induce fast splitting of a formed multiply charged vortex through, for example, the large asymmetric shape distortion [17]. Such splitting is considered to be one possible explanation for the short lifetime of the vortex observed in our experiment.

If the radial trap frequency $\omega_{r}$ is the same, the gravitational sag is independent of the atomic mass. On the other hand, the size of the BEC is estimated to be proportional to $m^{-1 / 2}$ under the Thomas-Fermi approximation. Therefore, the ratio between the gravitational sag and the BEC size is about 1.9 times larger for ${ }^{87} \mathrm{Rb}$ than for ${ }^{23} \mathrm{Na}$. This difference in the $\mathrm{BEC}$ size results in the large difference in the vortex formation between them, because the distance $d_{C M}$ allowed for the successful vortex formation should be smaller as the BEC size becomes smaller, while if the trap potential is the same, the gravitational sag depends on the atomic mass. When $B_{0}=0.4 \mathrm{G}$, the gravitational sag for $\mathrm{Na}$ was estimated to be $0.5 \mu \mathrm{m}$ in our magnetic trap, which was about one quarter of the value of $1.9 \mu \mathrm{m}$ for $\mathrm{Rb}$, and was much smaller than the typical radial width of the $\mathrm{Na}$ BEC. In the case of the field reversal from 0.4 to $-0.2 \mathrm{G}$ in a time more than $1 \mathrm{~ms}$, the distance $d_{C M}$ for $\mathrm{Na}$ was also estimated to be less than $0.05 \mu \mathrm{m}$ (see Fig. 7), which was about five times smaller than that for $\mathrm{Rb}$ and was much smaller than the typical radial width. Therefore, in the case of $\mathrm{Na}$, the gravitational sag does not strongly affect the vortex formation, which can explain the experimental difference between $\mathrm{Na}$ and $\mathrm{Rb}$.

\section{COMPENSATION OF GRAVITATIONAL FIELD}

From the discussion in the previous section, we expect that the vortex formation can be stabilized by compensating the gravitational field. For this purpose we applied on the BEC the optical dipole force generated by a far-blue-detuned laser beam (with wavelength $532 \mathrm{~nm}$ and the total power $0.75 \mathrm{~W}$ ). The nearly Gaussian laser beam was directed along the magnetic symmetry axis and was softly focused (with beam waist $\sim 53 \mu \mathrm{m}$ ) on the position about $54 \mu \mathrm{m}$ below the BEC (Fig. 8). The peak of the optical dipole force potential was $6.3 \mu \mathrm{K}$, and the vertical gradient around the BEC 


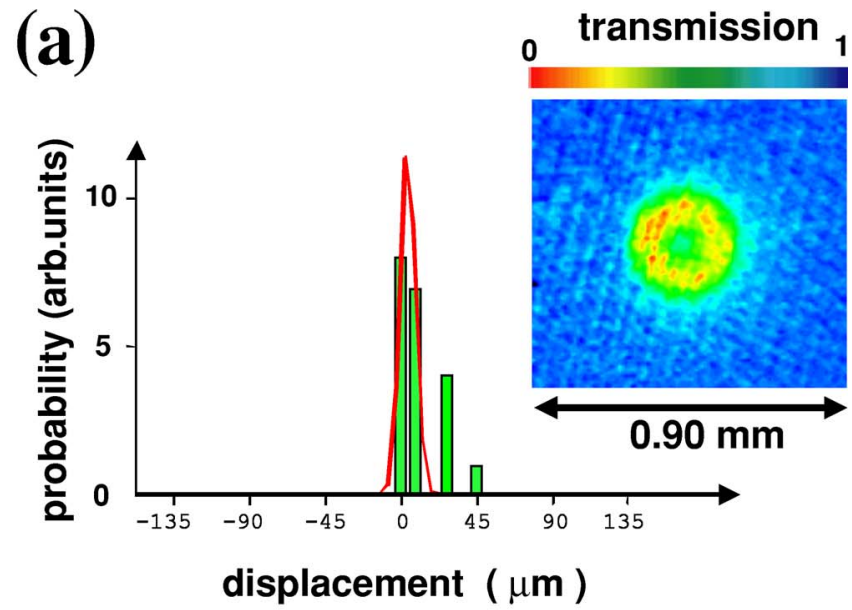

(b)

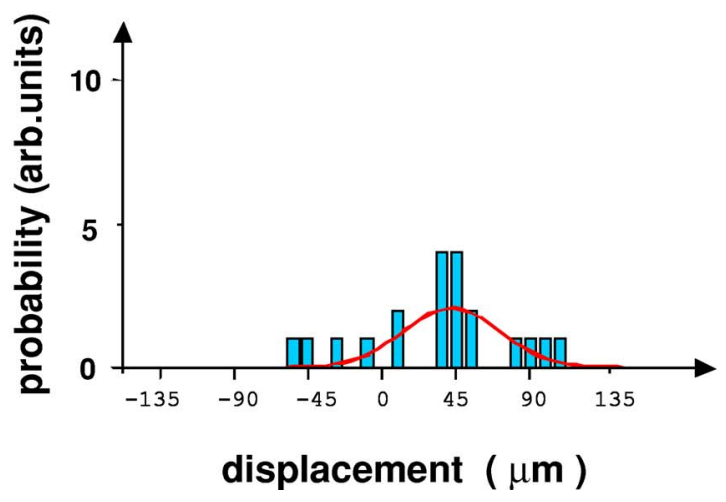

FIG. 9. (Color online) Probability distribution for the vertical displacement of the vortex center from the BEC center. (a) and (b) were obtained, respectively, with and without the gravity compensation. The axial magnetic field was reversed from $B_{0}=0.4$ to $-0.4 \mathrm{G}$ in a time of $3 \mathrm{~ms}$. The displacement was measured in the direction of the gravity. The inset presented in (a) is the image of the BEC with a vortex, which was observed along the $z$ direction with the gravity compensation ( $\mathrm{TOF}=25 \mathrm{~ms}$ ). The solid lines in (a) and (b) are Gaussian curves fitted to the distributions.

was compatible to the gravity force $-m g$. The compensation was confirmed by observing the freefall of the BEC with and without the compensation laser beam. The compensation beam was introduced during the rf evaporation cooling. So, the CM of the BEC always should be on the magnetic symmetry axis.

The quantum vortex was topologically formed by reversing the axial magnetic field in the same way as described in Sec. II. The created vortex was observed by monitoring the absorption image after the TOF, which was started by simultaneously turning off the magnetic trap and the compensation beam. The displacement between the centers of the vortex and the BEC was monitored. The probability distribution obtained is shown in Fig. 9, from which we see that, with the compensation laser beam, we have considerably improved the experimental stability. The width of the distribution was much smaller than that without the compensation beam, and the position of the vortex was nearly at the center of the
BEC. By fitting the distribution to a Gaussian function, we estimated the mean displacement and variance of the distribution. The mean displacement with and without the compensation were 4.3 and $42 \mu \mathrm{m}$, respectively, the improvement being about ten times. The variances were also improved from 41 to $6.2 \mu \mathrm{m}$ by the compensation.

The holding time of the BEC in which we could observe the vortex was elongated to $4 \mathrm{~ms}$, which was about four times the value without the gravity compensation. The vortex gradually diminished as the holding time became longer, and we could not observe the splitting of the vortex. One possible reason for the disappearance of the vortex in such a short holding time is the change of the structure of the vortex line. If the vortex line bent or vibrated in the BEC, we could not observe the vortex as a circular depletion in the atom density, because we observe the absorption image of the BEC by projecting along the $z$ direction. So, we consider that this holding time does not necessarily indicate the lifetime of the vortex.

The axial magnetic field after the field reversal probably affects the dynamics of the vortex also. After the reversal the magnetic field works as an antitrapping potential so that the BEC expands along the axial direction. To eliminate this effect, Shin et al. [18] inverted the axial potential curvature to increase vortex lifetime. Our holding time of $4 \mathrm{~ms}$ is much shorter than their values, but it might be necessary to remove or diminish the axial expansion to increase the vortex lifetime. The suppression of the expansion is essentially required for realizing much longer vortex lifetime, because the magnetic field after the reversal has two zero-field points on the $z$ axis. For a holding time within $4 \mathrm{~ms}$ the BEC cannot reach these points, which are separated more than $0.4 \mathrm{~mm}$ from the BEC center. However, for a holding time more than $20 \mathrm{~ms}$ the BEC can reach these points and these singularities should play an important role in the dynamics of the BEC.

\section{CONCLUSION}

We have successfully created the quadruply charged vortex in the Rb BEC with the topological method. From the experiment with $\mathrm{Rb}$ we have found that the vortex is formed when the experimental parameters such as $T_{\text {rev }}$ and $B_{0}$ were in narrow windows. The windows were much narrower than the case of $\mathrm{Na}$. To understand the existence of the windows we discussed at first the influence of the gravity on the vortex formation through the numerical calculation, and found that the vortex formation of heavy atoms was strongly affected by the gravitational sag and the vertical oscillation during the field reversal. We have also shown that the influence of the gravity can be removed by compensating the gravitational sag with the optical dipole force created by the blue-detuned laser beam aligned just below the BEC. The effect of the gravity may also be eliminated by orientating the $z$ axis vertically [26], but evaporative cooling in this configuration becomes more difficult.

The experimental stability in the vortex formation could be considerably improved by compensating the gravity. However, the holding time was about $4 \mathrm{~ms}$, which was considered still to be too short to observe the splitting of the 
multiply charged vortex. In the present experiment we have observed the vortex with a projectional method, but this method is not appropriate for monitoring a vortex whose vortex line bends or tilts. Tomography is one promising method for avoiding such possibilities to observe the splitting of the vortex, and we are now preliminarily trying to observe the splitting. To increase the vortex lifetime itself, it is probably necessary to suppress the axial expansion of the BEC after the field reversal, and we are also trying to diminish the expansion by using an optical dipole force trap.

To apply the multiply charged vortex to various quantummechanical researches, such as low-temperature physics, superfluidity, and quantum information technology, it is important to prolong the holding time without the splitting. To realize such an experimental system, optical pinning of the vortex using a optical plug [25] is one of the most powerful candidates for preventing the splitting.

\section{ACKNOWLEDGMENTS}

We thank T. Ohmi, Y. Kawaguchi, and M. Nakahara for useful discussions. We acknowledge financial support by MATSUO Foundation. This work was supported by the Grant-in-Aid for Scientific Research (Grant No. 13740255 and Grant No. 16540357) and the Grant-in-Aid for the 21st century COE "Center for Diversity and Universality in Physics" from the Ministry of Education, Culture, Sports, Science and Technology (MEXT) of Japan.
[1] L. P. Pitaevskii and S. Stringari, Bose-Einstein Condensation (Oxford University Press, Oxford, 2003).

[2] R. J. Donnelly, Quantized Vortices in Helium II (Cambridge University Press, Cambridge, 1991).

[3] M. Tinkham, Introduction to Superconductivity (MacGrawHill, New York, 1996).

[4] Bose-Einstein Condensation in Atomic Gases, edited by M. Inguscio, S. Stringari, and C. E. Wieman (IOS, Amsterdam, 1999).

[5] M. R. Matthews, B. P. Anderson, P. C. Haljan, D. S. Hall, C. E. Wieman, and E. A. Cornell, Phys. Rev. Lett. 83, 2498 (1999).

[6] K. W. Madison, F. Chevy, W. Wohlleben, and J. Dalibard, Phys. Rev. Lett. 84, 806 (2000).

[7] J. R. Abo-Shaeer, C. Raman, J. M. Vogels, and W. Ketterle, Science 292, 476 (2001).

[8] E. Hodby, G. Hechenblaikner, S. A. Hopkins, O. M. Maragò, and C. J. Foot, Phys. Rev. Lett. 88, 010405 (2001).

[9] P. C. Haljan, I. Coddington, P. Engels, and E. A. Cornell, Phys. Rev. Lett. 87, 210403 (2001).

[10] P. Engels, I. Coddington, P. C. Haljan, and E. A. Cornell, Phys. Rev. Lett. 89, 100403 (2002).

[11] P. Engels, I. Coddington, P. C. Haljan, V. Schweikhard, and E. A. Cornell, Phys. Rev. Lett. 90, 170405 (2003).

[12] S. Inouye, S. Gupta, T. Rosenband, A. P. Chikkatur, A. Görlitz, T. L. Gustavson, A. E. Leanhardt, D. E. Pritchard, and W. Ketterle, Phys. Rev. Lett. 87, 080402 (2001); F. Chevy, K. W. Madison, and J. Dalibard, ibid. 85, 2223 (2002); P. Rosenbusch, V. Bretin, and J. Dalibard, ibid. 89, 200403 (2002).

[13] M. Nakahara, T. Isoshima, K. Machida, S. Ogawa, and T. Ohmi, Physica B 284-288, 17 (2000); T. Isoshima, M. Nakahara, T. Ohmi, and K. Machida, Phys. Rev. A 61, 063610 (2000); S. I. Ogawa, M. Möttönen, M. Nakahara, T. Ohmi, and H. Shimada, ibid. 66, 013617 (2002); M. Möttönen, N. Matsumoto, M. Nakahara, and T. Ohmi, J. Phys.: Condens. Matter
14, 13481 (2002).

[14] A. E. Leanhardt, A. Görlitz, A. P. Chikkatur, D. Kielpinski, Y. Shin, D. E. Pritchard, and W. Ketterle, Phys. Rev. Lett. 89, 190403 (2002).

[15] A. E. Leanhardt, Y. Shin, D. Kielpinski, D. E. Pritchard, and W. Ketterle, Phys. Rev. Lett. 90, 140403 (2003).

[16] P. Nozières and D. Pines, Superfluid Bose Liquids (AddisonWesley, Reading, MA, 1990).

[17] Y. Kawaguchi and T. Ohmi, Phys. Rev. A 70, 043610 (2004).

[18] Y. Shin, M. Saba, M. Vengalattore, T. A. Pasquini, C. Sanner, A. E. Leanhardt, M. Prentiss, D. E. Pritchard, and W. Ketterle, Phys. Rev. Lett. 93, 160406 (2004).

[19] D. S. Hall, M. R. Matthews, J. R. Ensher, C. E. Wieman, and E. A. Cornell, Phys. Rev. Lett. 81, 1539 (1998).

[20] M. Kumakura, Y. Shirahata, Y. Takasu, Y. Takahashi, and T. Yabuzaki, Phys. Rev. A 68, 021401 (R) (2003); K. Toyoda, Y. Takahashi, and T. Yabuzaki, J. Phys. Soc. Jpn. 71, 1445 (2002).

[21] C. J. Myatt, N. R. Newbury, R. W. Ghrist, S. Loutzenhiser, and C. E. Wieman, Opt. Lett. 21, 290 (1996).

[22] M.-O. Mewes, M. R. Andrews, N. J. van Druten, D. M. Kurn, D. S. Durfee, and W. Ketterle, Phys. Rev. Lett. 77, 416 (1996).

[23] E. Lundh, C. J. Pethick, and H. Smith, Phys. Rev. A 58, 4816 (1998); F. Dalfovo and M. Modugno, ibid. 61, 023605 (2000).

[24] Y. Kawaguchi, M. Nakahara, and T. Ohmi, Phys. Rev. A 70, 043605 (2004).

[25] K. B. Davis, M. -O. Mewes, M. R. Andrews, N. J. van Druten, D. S. Durfee, D. M. Kurn, and W. Ketterle, Phys. Rev. Lett. 75, 3969 (1995).

[26] D. G. Fried, T. C. Killian, L. Willmann, D. Landhuis, S. C. Moss, D. Kleppner, and T. J. Greytak, Phys. Rev. Lett. 81, 3811 (1998); A. E. Leanhardt, T. A. Pasquini, M. Saba, A. Schirotzek, Y. Shin, D. Kielpinski, D. E. Pritchard, and W. Ketterle, Science 301, 1513 (2003). 